\title{
Expression and translocation of chlamydial protease during acute and persistent infection of the epithelial HEp-2 cells with Chlamydophila (Chlamydia) pneumoniae
}

Dagmar Heuer, Volker Brinkmann, Thomas F. Meyer and Agnes J. Szczepek ${ }^{\star}$

Max-Planck Institute for Infection Biology, Department Molecular Biology, Schumannstr. 21/22, 10117 Berlin, Germany.

\section{Summary}

Chlamydial protease-like activity factor (CPAF) is secreted to the cytoplasm of the infected cells where it proteolytically cleaves eukaryotic transcription factor RFX5. Here, we determined the localization pattern of CPAF during the course of an acute and persistent in vitro infection of the epithelial cell line HEp-2 with Chlamydophila pneumoniae strain VR1310. Using immunoblotting, confocal microscopy and electron microscopy, we found CPAF in the inclusion lumen or associated with bacteria during the first $48 \mathrm{~h}$ of an acute infection. Seventy-two hours and later, CPAF was present predominantly in the cytoplasm of the infected cells. Translocation of CPAF into cytoplasm correlated in time with degradation of the transcription factor RFX5, as confirmed by immunoblotting. Interestingly, during the persistent infection induced by either IFN- $\gamma$ or iron limitation CPAF translocation to the cytoplasm was inhibited resulting in unaffected or only partially reduced levels of RFX5. Based on presented findings, we propose that CPAF translocation to the cytoplasm is separated from its production. The translocation mechanism appears to be fully active during an acute infection; however, it is fully or partially inhibited during persistent infection induced by IFN- $\gamma$ or by iron limitation respectively. Consequently, this work demonstrates the importance of subcellular localization of CPAF for the characteristics of chlamydial acute and persistent infection in epithelial HEp-2 cells.

Received 24 September, 2002; revised15 January, 2003; accepted 22 January, 2003. *For correspondence. E-mail agnes@mpiib-berlin.mpg.de; $\quad$ Tel. $\quad$ (+49) 3028460 426; $\quad$ Fax (+49) 3028460401.

(c) 2003 Blackwell Publishing Ltd
Introduction

The number of the bacterial proteins that directly interact with eukaryotic targets is increasing (Naumann et al., 1999; Galan, 2001; Kresse et al., 2001). Recently cloned and characterized chlamydial proteases encoded by Ct858 in Chlamydia trachomatis and Cpn1016 in Chlamydophila pneumoniae genomes are the first chlamydial proteins that were shown to be secreted to the cytoplasm of the infected cell (Zhong et al., 2001; Fan et al., 2002; Shaw et al., 2002). Despite low homology on the amino acid level, both proteases target the same eukaryotic transcription factor RFX5 required for the expression of both classes of MHC (van den Elsen et al., 1998; Gobin et al., 2001). Consequently, the presence of active CPAF in the cytoplasm of the infected epithelial cell leads to downregulation of the host adaptive immune defence (Zhong et al., 1999; 2000).

Importantly for the medical outcome of chlamydial infections, both $C$. trachomatis and $C$. pneumoniae are capable of causing persistent infections in vivo (Hammerschlag et al., 1992; Dean et al., 2000). During persistent infection, $C$. trachomatis and $C$. pneumoniae do not undergo their typical developmental cycle as defined by consecutive presence of two forms, elementary and reticulate bodies (EB and RB respectively) but rather develop into an intermediate, non-infectious and non-replicating form, characterized by aberrantly enlarged size and altered metabolic activity (Beatty et al., 1993; Al Younes et al., 2001). These intermediate aberrant chlamydial forms were reported to persist in the infected cells for a time longer than needed to complete a normal developmental cycle (Beatty et al., 1995). Interestingly, it was also reported that during the persistent infection induced by IFN- $\gamma$, cells infected with $C$. trachomatis retained some properties previously demonstrated in the acute infection, specifically the inhibition of apoptosis (Dean and Powers, 2001).

To understand the mechanism of in vivo persistency, several in vitro models of persistent infections were developed. One of the most widely used is the persistency induced by treatment with IFN- $\gamma$, which reflects the in vivo inflammatory response of infected animal or individual 
(Beatty et al., 1993; Mehta et al., 1998). The mechanism behind IFN- $\gamma$-induced persistency was shown to depend on reversible modulation of tryptophan availability in the infected cell by the induction of tryptophan-catabolizing enzyme indoleamine 2,3-dioxygenase (Beatty et al., 1995). In another model, that has recently been established in our laboratory, persistent state of infection was induced by limiting availability of iron (Al Younes et al., 2001). The precise mechanism regulating that type of persistency is still unknown. However, earlier studies showed that iron concentration affects the infectivity and length of developmental cycle for both, $C$. trachomatis and C. pneumoniae (Raulston, 1997; Al Younes et al., 2001). Additionally, increased iron levels were previously indicated as a risk factor connected with chlamydial presence in atherosclerosis (Sullivan and Weinberg, 1999).

Chlamydial protease-like activity factor appears to modulate the acquired immune response against chlamydial infections by proteolysis of RFX5 transcription factor. Thus, we felt that it would be important to study when and under which conditions CPAF is expressed and active within the host cell. Our goal was to explore the kinetics of CPAF expression during an acute infection and to determine its localization at different stages of the chlamydial developmental cycle. We also wanted to determine the influence of a persistent infection on the expression and localization of CPAF. To gain a broader view on this subject, we compared two different models of persistent infections induced by IFN- $\gamma$ or by iron limitation.

\section{Results}

C. pneumoniae expresses CPAF at $24 \mathrm{~h}$ post infection; however, CPAF is translocated to cytoplasm later than 48 h post infection

To study the expression of CPAF during the course of infection, the levels of CPAF were monitored by immunoblotting. Using mouse monoclonal antibody against CPAF, we detected the characteristic double band (35 and $37 \mathrm{kDa}$ ) (Fig. 1). On days 2 and 3 post infection (p.i.), both isoforms were expressed whereas on day 4 p.i., the predominant isoform was $35 \mathrm{kDa}$. Additionally, we performed immunostaining of the infected cells 1, 2, 3 and 4 days p.i. Using confocal microscopy, we observed a weak staining inside some of the inclusions on day 1 (data not shown) followed by a strong staining in the majority of the inclusions on day 2 (Fig. 2A). During first two days of infection, CPAF was localized predominantly inside of the inclusions. On days 3 and 4 p.i., CPAF was detected mainly in the cytoplasm of the infected cells, indicative of its translocation (Fig. 2B and C). Two staining patterns were identified: first, diffused throughout the cytoplasm and a second, vesicular-like.

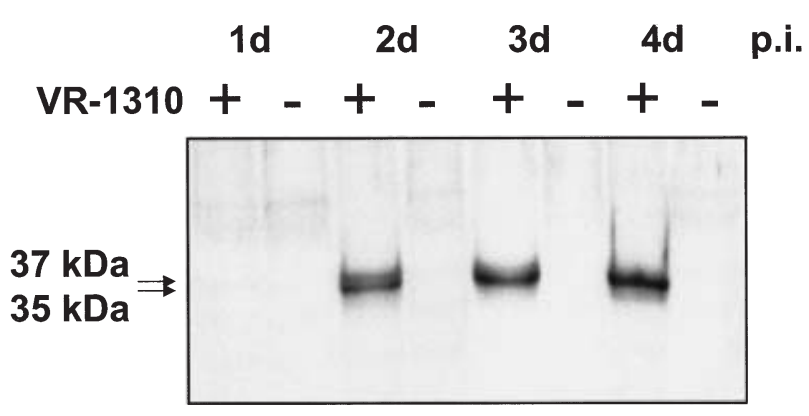

Fig. 1. CPAF expression pattern during the course of an acute infection - detection of CPAF by immunoblotting. HEp-2 cells were infected with C. pneumoniae strain VR-1310. After incubation for 1, 2, 3 or 4 days, the cells were lysed and proteins were separated on a $10 \%$ SDS gel. After electroblotting, CPAF was detected using mouse monoclonal antibody EB 3.1 followed by goat anti-mouse-HRP and visualized using chemiluminescence system. Using this method, CPAF could be detected on the second day p.i. Two bands, 35 and $37 \mathrm{kDa}$ (indicated by arrows on the blot) were present on day 2 and 3 p.i. whereas only one, $35 \mathrm{kDa}$ predominant band was present at the end of the infectious cycle.

Presence of CPAF in the cytoplasm of infected HEp-2 cells correlates with the downregulation of RFX5

The mammalian transcription factor RFX5 was found earlier to be a target for the proteolytic activity of CPAF. To establish at what time after infection RFX5 is degraded, the infected cells were collected on daily intervals and subjected to SDS-PAGE and immunoblotting with an antiRFX5 antibody. We observed a degradation of RFX5 on days 3 and 4 p.i., as compared to uninfected controls (Fig. 3). The degradation of RFX5 correlated in time with translocation of CPAF into the cytoplasm.

\section{Electron microscopy studies confirm early localization of CPAF to the chlamydial inclusion}

To more accurately determine the localization of CPAF, we performed immunogold EM studies. The staining was performed on the second (Fig. 4A) and fourth (Fig. 4B) day p.i., as described in Experimental procedures. Respective controls (uninfected cells, infected cells incubated with either primary or secondary antibodies only) returned negative results confirming the immunoblotting and immunofluorescence control staining and indicating high specificity of the EB3.1 antibody (data not shown). We observed that on day 2 p.i. CPAF was localized in the inclusion associated with bacteria. The gold particles were also associated with small vesicular structures that could either be a topical part of the $C$. pneumoniae outer membrane or a separate vesicle of unknown nature (Fig. 4A). In the staining performed on day 4 post infection, we were unable to detect CPAF in the inclusion (Fig. 4B). 

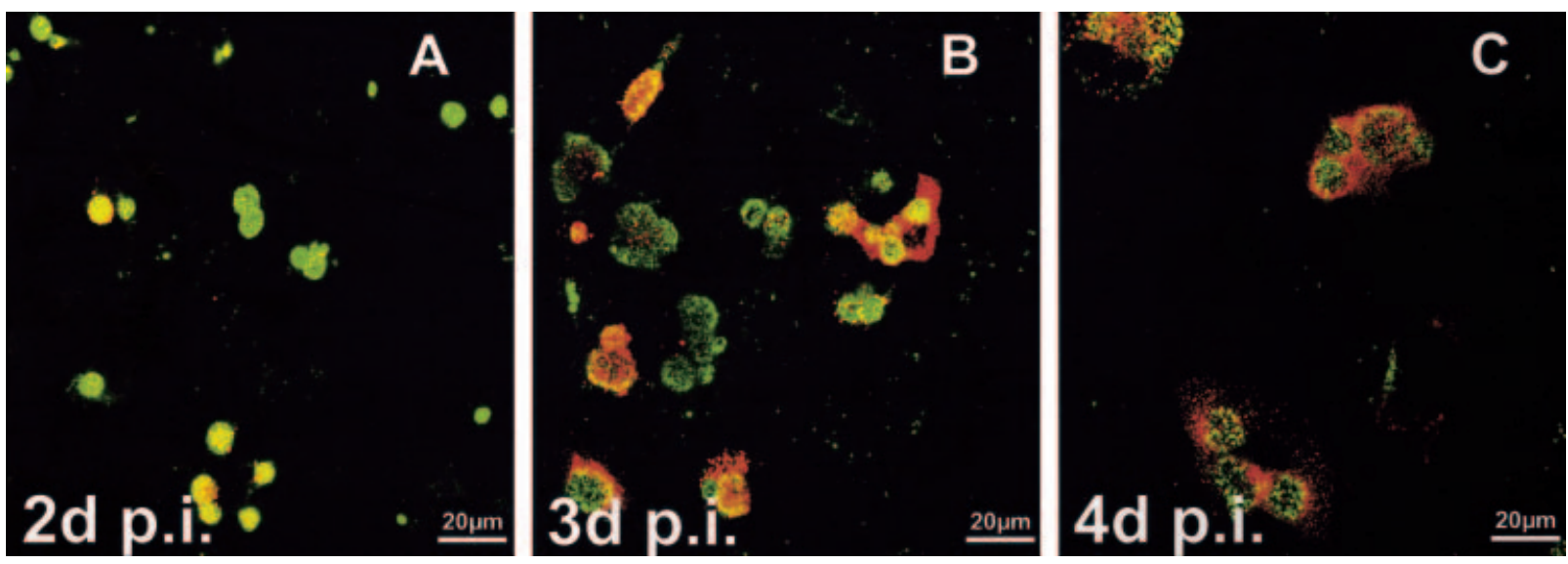

Fig. 2. Localization of CPAF during an acute infection - immunofluorescence studies. HEp-2 cells were infected with VR-1310 (MOI = 1) and incubated for 2, 3 and 4 days. Next, the cells were washed, fixed with 2\% PFA and stained using monoclonal antibody against CPAF EB3.1 (Cy3) and polyclonal rabbit sera raised against genus-specific chlamydial antigens (Cy2). Shown are micrographs from the confocal microscope. A. 2 days p.i. CPAF (red) is localized inside the inclusions and fully co-localized with chlamydial genus-specific antigen (green), as indicated by yellow staining resulting from the overlap.

B. 3 days p.i. CPAF translocates into the cytoplasm but is still present in some of the inclusions.

C. 4 days p.i. CPAF is predominantly present in the cytoplasm and on the borders of the inclusions.

\section{In the persistent infections, CPAF does not translocate into cytoplasm of the infected cell}

Chlamydial infections are often of persistent nature in vivo. To date, at least four models of the in vitro persistent infections were established, namely induction of persistency by addition of IFN- $\gamma$, addition of antibiotics, nutrient depletion and iron depletion. To observe expression and localization of CPAF during the course of persistent infection, we chose to use the addition of IFN- $\gamma$ or iron depletion as two different inducers of persistency. Fifty units of IFN$\gamma \mathrm{ml}^{-1}$ or $30 \mu \mathrm{M}$ DAM were added to the infected cultures 1 day or $4 \mathrm{~h}$ after the beginning of the infection respectively. The infection was monitored for up to 6 days. Using confocal microscopy, we observed that CPAF is produced by C. pneumoniae in both types of persistent infections. On day 3 p.i., where in control infected cells majority of CPAF

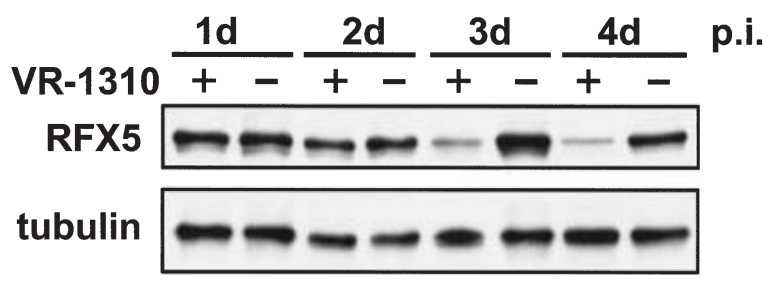

Fig. 3. RFX5 mammalian transcription factor is degraded on days 3 and 4 during the course of an acute infection. Cells were infected with VR-1310 ( $\mathrm{MOI}=1$, without cycloheximide). At indicated time points, the cells were lysed in RIPA buffer and the proteins were separated by $10 \%$ SDS-PAGE. After electroblotting, RFX5 was detected using rabbit polyclonal antibody against RFX5 followed by a HRPconjugated secondary antibody and visualized by ECL reaction. Antitubulin staining was used for standardization of the blot. The degradation of RFX5 is seen on days 3 and 4 p.i., but not the earlier times. is translocated into the cytoplasm, in the persistently infected cells CPAF was predominantly localized inside of the inclusions (Fig. 5A). In the persistently infected cells, this localization remained unchanged up to 6 days p.i. In the acute infection 6 days p.i. corresponds to second developmental cycle, thus this time point could not be analysed. Interestingly, in the DAM-treated samples, unlike in the IFN- $\gamma$-treated ones we observed occasionally CPAF staining in small cytoplasmic vesicles (Fig. 5B, arrows).

\section{Absence of translocated CPAF in the cytoplasm correlates with unaffected levels of RFX5}

As the translocation of CPAF appeared to be inhibited in both types of persistent infections, we wanted to examine the levels of RFX5 under the persistent conditions. To do that, we detected RFX5 in an immunoblot of the cells infected with $C$. pneumoniae and treated with IFN- $\gamma$ or DAM. Our results demonstrated that despite of the production of CPAF by C. pneumoniae, the levels of RFX5 were unchanged in the IFN- $\gamma$-treated cultures and decreased, but not eradicated, in the DAM-treated cultures, as compared to the respective controls (Fig. 6).

\section{Discussion}

Chlamydial protease-like activity factor is the first chlamydial protein unequivocally shown to be translocated into host cell cytoplasm where it specifically targets transcription factors resulting in impairment of the immune system (Zhong et al., 2000; 2001; Fan et al., 2002). In this work, we demonstrated that CPAF was produced by C. pneumoniae from day 1 p.i. which is in agreement with the $2 \mathrm{D}$ 

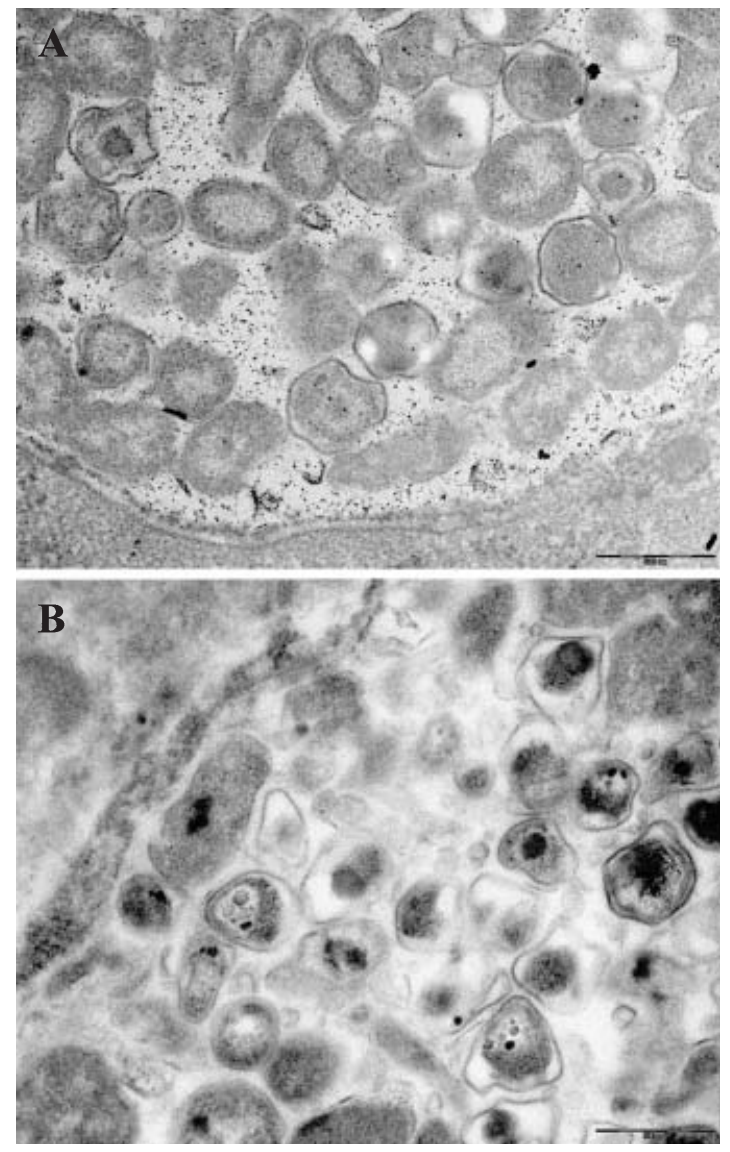

Fig. 4. CPAF is localized in the chlamydial inclusion 2 but not 4 days after infection. EM immunogold labelling. Infected cells treated as described in Experimental procedures. Ultrathin sections were incubated with the mouse monoclonal antibody EB3.1 followed by a goat anti-mouse antibody coupled to $1 \mathrm{~nm}$ gold colloids. For the evaluation of staining, a Leo906 transmission electron microscope was used. A. Demonstrates a specimen 2 days p.i. Gold particles indicate presence of CPAF localized inside of the inclusion and either associated with bacteria or present in the lumen of the inclusion (arrows). B. Shows specimen that was infected for 4 days. No CPAF could be detected inside of the inclusion. The bar in the right-side lower corner of the micrographs indicates $200 \mathrm{~nm}$ size.

data obtained by Shaw et al. (2002). We also determined that during the first 2 days of infection, CPAF remained within the chlamydial inclusion. At later time points, we observed translocation of CPAF into cytoplasm of the infected cells. The translocation correlated in time with the degradation of RFX5, which is substrate protein for CPAF. However, it cannot be excluded that the translocation takes place earlier than 3 days p.i. but could not be appreciated as a result of the limited sensitivity of assays used to trace both CPAF and RFX5. Chlamydophila pneumoniae is well known to cause persistent infections during which chlamydial metabolism is altered as compared to the acute infection. Thus, we investigated the expression and localization pattern of CPAF during two distinct types of persistent infections. In both of them, the one induced by addition of IFN- $\gamma$ which mimics inflammatory host response and the other caused by the depletion of iron corresponding to a medical condition of anaemia, we observed that CPAF was indeed produced by $C$. pneumoniae yet it was retained within inclusions. The full or a partial inhibition of CPAF translocation correlated with stable levels of RFX5 in the IFN- $\gamma$-treated cultures or with decreased levels of RFX5 in the DAM-treated cultures, respectively. Only partially decreased amount of RFX5 in the DAM-treated cultures could be attributed to the occasional presence of cytoplasmic vesicles containing CPAF.

Our findings suggest that the expression and translocation of CPAF to the cytoplasm of infected cells are not simultaneous. Moreover, we demonstrated that CPAF undergoes post-translational processing. The mouse monoclonal antibody EB 3.1 that we used throughout this work, was raised against the C-terminal portion of CPAF and in the immunoblotting it has never detected full length CPAF $(70 \mathrm{kDa})$, corroborating the observations of others (Fan et al., 2002; Shaw et al., 2002). Instead, this antibody visualized two bands ( 35 and $37 \mathrm{kDa}$ ) indicative of either two distinct cleavage sites or other postranslational modification, i.e. phosphorylation. Interestingly, the two isoforms were not expressed on equal levels throughout the infection. The $37 \mathrm{kDa}$ and $35 \mathrm{kDa}$ isoforms were produced during the first stages of an acute infection and throughout the duration of the persistent infection (data not shown). The $35 \mathrm{kDa}$ isoform was predominant at the end of the acute infection. The nature of these modifications remains to be clarified. Zhong et al. (2001) have shown that the recombinant full length CPAF from $C$. trachomatis retained its proteolytic activity, therefore the

Fig. 5. Inhibition of CPAF translocation during persistent infection induced by IFN $\gamma$ or DAM.

A. Inhibition of CPAF translocation in infected cells treated with IFN $\gamma$ and DAM 3 days post infection. To induce persistent infection, the cells were infected and after 1 day of incubation, $50 \mathrm{U} \mathrm{ml}^{-1} \mathrm{IFN} \gamma$ was added to the cells. Alternatively, the cells were infected and incubated for $4 \mathrm{~h}$ under standard conditions followed by the addition of $30 \mu \mathrm{M}$ DAM and $\mathrm{CHX}$. Infected control cells remained untreated. Three days p.i. the cells were fixed and CPAF was detected using mouse monoclonal EB3.1 (Cy3). Inclusions were visualized using a genus-specific rabbit polyclonal antibody (Cy2). Shown are micrographs from the confocal microscope. Right panel shows overlay of channels Cy2 and Cy3 with the contrast phase. Unlike in the control infected untreated cells, in the persistent infection induced by either IFN $\gamma$ or DAM, CPAF is predominantly present inside of the inclusions.

B. Inhibition of CPAF translocation in infected cells treated with IFN $\gamma$ and DAM 6 days post infection. Cells were treated as described above. Six days p.i. the persistently infected cells were fixed and CPAF was detected using mouse monoclonal antibody EB3.1 (Cy3). Inclusions were visualized using polyclonal rabbit sera raised against genus-specific chlamydial antigens (Cy2). Shown are micrographs from the confocal microscope. In the DAM-treated cells, CPAF is present inside of the inclusions but also shows vesicular distribution in some cells (arrows). 

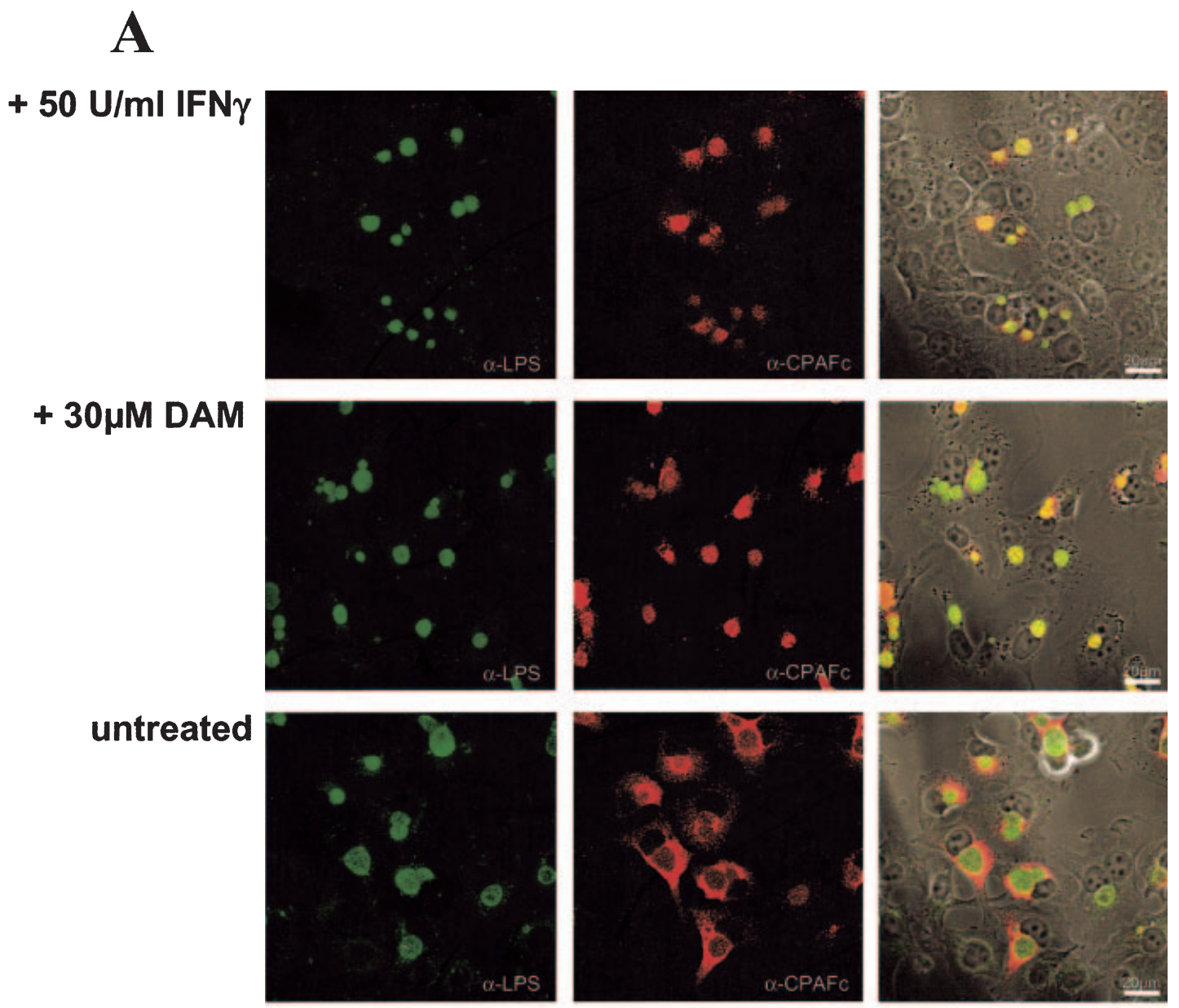

B

\section{$+50 \mathrm{U} / \mathrm{ml} \mathrm{IFN} \gamma$}
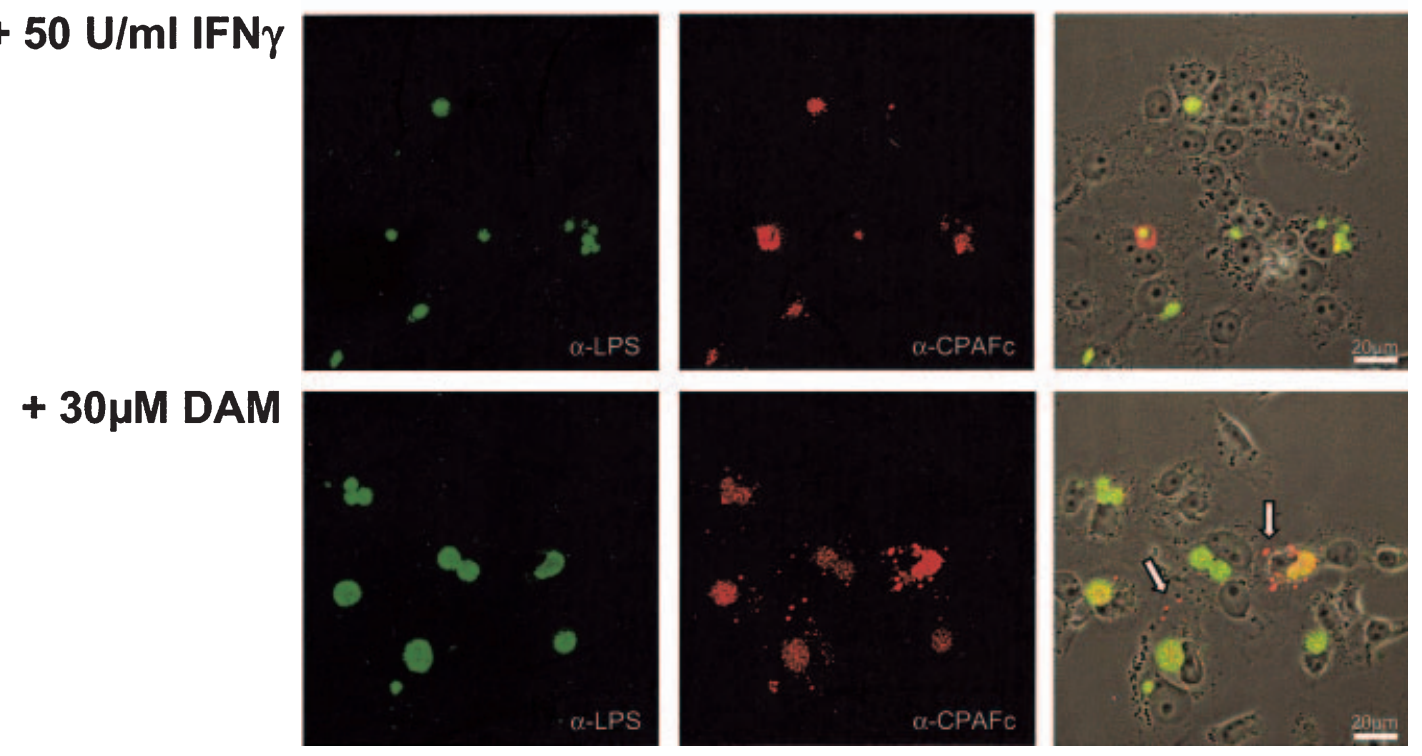


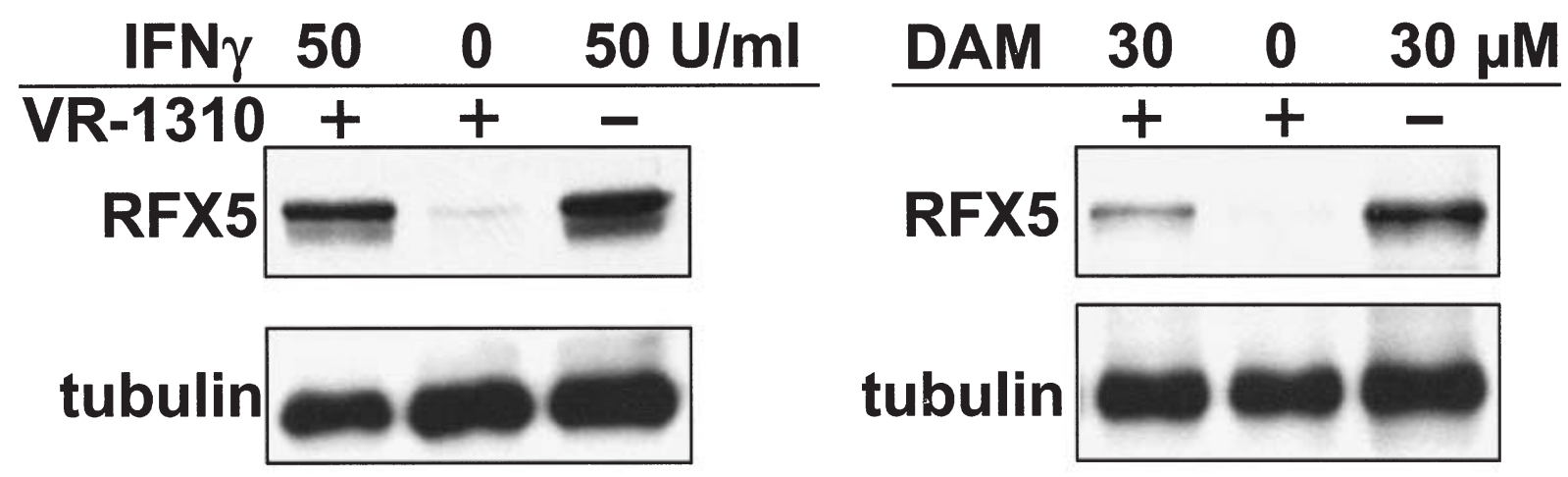

Fig. 6. Degradation of RFX5 is fully or partially inhibited in persistent infections. HEp-2 ells were infected and the persistence was induced as described in Experimental procedures. Three days p.i. the medium was replaced with medium containing no cycloheximide and the cells were incubated for an additional $24 \mathrm{~h}$. Next, RFX5 was detected in a cell lysate, as described in Experimental procedures. Tubulin staining was used as a loading control. Left panel demonstrates stable presence of RFX5 in the persistently infected cells exposed to IFN- $\gamma$. Right panel shows decreased but not abrogated levels of RFX5 in the DAM-treated infected cells. Both uninfected/treated and infected/untreated cells were used as a control.

purpose of CPAF cleavage needs to be explained. The mechanism of CPAF secretion by bacteria into the lumen of inclusion also remains to be elucidated. Although components of the type III secretion system have been identified in the genome and in the proteome of $C$. pneumoniae (Kalman et al., 1999; Vandahl et al., 2001), it is rather unlikely that this particular system would be involved in the transport of CPAF. We concluded this based on computer-assisted predicted localization of CPAF indicating presence of a signal peptide. The translocation mechanism into the cytoplasm of the infected cell is also unclear. The fact that in persistent infection, the translocation but not the production of CPAF was inhibited, suggests that the transport process is not self-mediated by CPAF. Additional factor(s) that are limited by the persistent conditions must play a role in this mechanism.

The proteolytic activity of CPAF, as measured by presence or absence of the transcription factor RFX5 in the infected cells, correlated with CPAF translocation that took place later than 2 days p.i. but not with CPAF production, detected already on day 1 p.i. Interestingly, as the CPAF translocation was inhibited in persistent infections, so was the degradation of RFX5. The persistent infections were induced in two different manners; however, both resulted in full or partial inhibition of CPAF translocation. Full inhibition was evident in the IFN- $\gamma$ treated cultures and correlated with intact levels of RFX5. In the DAM-treated cells; however, likely as a result of the occasional presence of cytoplasmic vesicular CPAF, the levels of RFX5 decreased. It is tempting to speculate that both, addition of IFN- $\gamma$ and iron depletion induce similar phenotypic changes on either host or bacterial side, as defined by the inhibition of CPAF translocation. Alternatively, addition of IFN- $\gamma$ and iron limitation could induce or affect distinct pathways resulting in comparable outcome.

Interesting aspect of the inhibition of CPAF translocation into the cytoplasm is its immune consequence. RFX5 is responsible for transcriptional upregulation of the MHC class II in response to IFN- $\gamma$ but also for a baseline expression of MHC class I (Reith and Mach, 2001). Thus, in persistent infections when the levels of RFX5 are unaffected, antigen presentation in context of both MHC classes should be normal or even upregulated. However, if mechanism of CPAF translocation is common for other chlamydial proteins, then lack of translocated CPAF in the cytoplasm during persistent infections would indicate no other chlamydial antigens available for presentation. Interestingly, there is evidence that even if such presentation would take place, activated T cells that recognize chlamydial antigens undergo apoptosis (Jendro et al., 2000). Moreover, epithelial cells persistently infected with $C$. trachomatis (IFN $\gamma$-induced persistence) resist apoptotsis (Dean and Powers, 2001) similarly to acute infection of epithelium with C. pneumoniae or C. trachomatis (Fan et al., 1998; Rajalingam et al., 2001). Taken together, multiple mechanisms protect the persistently infected cells from recognition by immune system and from the immune cytotoxic effector mechanisms.

Chlamydial protease-like activity factor is the first and so far unique chlamydial protein found to be translocated into the cytoplasm of the infected host cell. The motif and the homology searches within the genome and proteome of $C$. pneumoniae so far returned no hits indicative of existence of protein(s) similar either in function, structure or in processing (our unpublished study). Nevertheless, the mechanism behind the translocation of CPAF could theoretically be used for other chlamydial proteins. Consequently, inhibition of this mechanism during persistent 
infections would prevent the direct eukaryotic-prokaryotic interactions and also leave the chlamydial proteins inaccessible for immune processing and presentation, deep inside the chlamydial inclusions.

\section{Experimental procedures}

\section{Media}

Cell growth media consisted of RPMI 1640 (Invitrogen $\mathrm{GmbH}$, Karlsruhe, Germany) supplemented with $10 \%$ FBS (fetal bovine serum) (Biochrome KG, Berlin, Germany). For the infection, RPMI 1640 media was supplemented with 5\% FBS and occasionally with $1 \mu \mathrm{g} \mathrm{ml}^{-1}$ cycloheximide (CHX) (Sigma-Aldrich, Munich, Germany), as indicated for specific experiments.

\section{Infection protocol}

Hep-2 cells were seeded in 6-well plates with or without cover slips one day before infection. On the following day, the host cells were infected with VR1310 using specified MOI. Briefly, bacteria were centrifuged onto cells for $1 \mathrm{~h}$ at $33^{\circ} \mathrm{C}$ using $920 \mathrm{~g}$ and incubated under standard conditions $\left(35^{\circ} \mathrm{C}\right.$ and $5 \% \mathrm{CO}_{2}$ in a humidified tissue culture incubator) for indicated time in the infection media with or without $1 \mu \mathrm{g} \mathrm{ml}^{-1}$ cycloheximide.

\section{Induction of persistence with IFN- $\gamma$ or deferoxamine mesylate (DAM)}

To induce persistent infections, infected HEp-2 cells were treated either with IFN- $\gamma$ (Roche Diagnostics $\mathrm{GmbH}$, Mannheim, Germany) or with DAM (Sigma-Aldrich). In case of IFN- $\gamma$-induced persistency, HEp-2 cells were infected with an MOI of 1.5 and incubated in RPMI $1640 / 5 \%$ FBS for one day $\left(35^{\circ} \mathrm{C}\right.$ and $5 \% \mathrm{CO}_{2}$ in a humidified tissue culture incubator). Then the media was replaced with RPMI 1640/5\% FBS containing $50 \mathrm{U} \mathrm{ml}^{-1}$ IFN- $\gamma$ followed by incubation for up to 6 days under standard conditions. To induce persistency by iron depletion, HEp-2 cells were infected with an $\mathrm{MOI}$ of 1.5 . Four hours post infection (p.i) the inoculum was replaced by RPMI $/ 5 \%$ FBS supplemented with $1 \mu \mathrm{g} \mathrm{ml}^{-1}$ cycloheximide and $30 \mu \mathrm{M}$ DAM. The plates were incubated under standard conditions for specified times.

\section{Cell line, bacterial strains and bacterial propagation}

As a host cell, the larynx carcinoma cell line HEp-2 was used. Chlamydia pneumoniae strain CWL029 (ATCC strain VR1013, a kind gift from Dr Gunna Christiansen, University of Aarhus, Aarhus, Denmark) was propagated in HEp-2 larynx carcinoma cell line according to standard procedures $\left(35^{\circ} \mathrm{C}\right.$ and $5 \% \mathrm{CO}_{2}$ in a humidified tissue culture incubator). Presence of Mycoplasma sp. was excluded using PCR, mycoplasma detection system (Roche Diagnostics, Basel, Switzerland) and DAPI staining (Sigma-Aldrich).

\section{Antibodies, cell staining and immunoblot analyses}

The mouse monoclonal antibody EB3.1 against CPAF was a kind gift from Dr Guangming Zhong (University of Texas Health Sci- ence Center at San Antonio, San Antonio, TX). EB3.1 was supplied as a supernatant from a hybridoma cell line. Genus-specific rabbit polyclonal antibodies were from Milan Analytica (La Roche, Switzerland). Anti-RFX5 rabbit IgG fraction was purchased from Rockland Immunochemicals (Gilbertsville, PA). Mouse antitubulin antibody was from Sigma-Aldrich. Secondary antibodies coupled with fluorochromes were from Molecular Probes (Leiden, the Netherlands).

For the confocal microscopy analysis, on indicated days post infection, the cells were washed with PBS, fixed for 30 min with $2 \%$ paraformaldehyde (Sigma-Aldrich) and then stained with the respective antibodies (EB3.1 was used at a dilution of 1:50 or 1:100, depending on the preparation; standardization data not shown), as described previously (Al Younes et al., 2001). Western blotting was done according to standard procedures with Western Lightning ${ }^{\mathrm{TM}}$ Chemiluminescence Reagent detection system (Perkin Elmer Life Sciences, Boston, MA). EB3.1 was used in WB at a dilution of 1:500 or 1:1000, depending on the preparation (standardization data not shown).

\section{EM immunostaining}

Infected cells and uninfected controls were fixed in 4\% PFA/0.1\% GA (Sigma-Aldrich) in PBS for $20 \mathrm{~min}$ at room temperature and permeablilized with $0.1 \%$ saponin (Sigma-Aldrich) in PBS. After 30 min blocking ( $1 \% \mathrm{NGS} / 1 \% \mathrm{BSA}$ in PBS) the cells were incubated with the EB 3.1 anti-CPAF MoAb $\left(24 \mathrm{~h}\right.$ at $4{ }^{\circ} \mathrm{C}, 1: 100$ in PBS). Cells were washed and incubated with the secondary antibody coupled to $1 \mathrm{~nm}$ gold $\left(48 \mathrm{~h}\right.$ at $\left.4^{\circ} \mathrm{C}, 1: 100\right)$. After washing, gold colloids were visualized using silver enhancement (Danscher, 1981), cells were post-fixed $\left(0.5 \% \mathrm{OsO}_{4}\right.$ (Sigma-Aldrich)), dehydrated and embedded in Polybed 812 (Polysciences Europe, GmbH Eppelheim, Germany). After polymerization for 2 days at $65^{\circ} \mathrm{C}$, sections were cut at $60 \mathrm{~nm}$ and analysed using a LEO 906E (A Carl Zeiss SMT AG Company, UK) transmission electron microscope.

\section{Acknowledgements}

We would like to thank Dr Guangming Zhong for supplying us with the monoclonal antibodies against CPAF and for helpful discussions; Dr Gunna Christiansen for her generous gift of Mycoplasma sp.-free chlamydial strain VR1310. This work was supported in part by the Founds der Chemischen Industrie (to T.F.M.). We would like to thank Hesham M. Al-Younes, André Mäurer, Nicole Paland, Marion Rother, and Wolfgang Wehrl for critical reading of this manuscript and helpful discussions. Many thanks to Elke Ziska and Marco Kachler for their skilled technical assistance.

\section{References}

Al Younes, H.M., Rudel, T., Brinkmann, V., Szczepek, A.J., and Meyer, T.F. (2001) Low iron availability modulates the course of Chlamydia pneumoniae infection. Cell Microbiol 3: 427-437.

Beatty, W.L., Byrne, G.I., and Morrison, R.P. (1993) Morphologic and antigenic characterization of interferon gammamediated persistent Chlamydia trachomatis infection in vitro. Proc Natl Acad Sci USA 90: 3998-4002. 
Beatty, W.L., Morrison, R.P., and Byrne, G.I. (1995) Reactivation of persistent Chlamydia trachomatis infection in cell culture. Infect Immun 63: 199-205.

Danscher, G. (1981) Localization of gold in biological tissue. A photochemical method for light and electronmicroscopy. Histochemistry 71: 81-88.

Dean, D., and Powers, V.C. (2001) Persistent Chlamydia trachomatis infections resist apoptotic stimuli. Infect Immun 69: 2442-2447.

Dean, D., Suchland, R.J., and Stamm, W.E. (2000) Evidence for long-term cervical persistence of Chlamydia trachomatis by omp1 genotyping. J Infect Dis 182: 909-916.

van den Elsen, P.J., Gobin, S.J., van Eggermond, M.C., and Peijnenburg, A. (1998) Regulation of MHC class I and II gene transcription: differences and similarities. Immunogenetics 48: 208-221.

Fan, P., Dong, F., Huang, Y., and Zhong, G. (2002) Chlamydia pneumoniae secretion of a protease-like activity factor for degrading host cell transcription factors required for major histocompatibility complex antigen expression. Infect Immun 70: 345-349.

Fan, T., Lu, H., Hu, H., Shi, L., McClarty, G.A., Nance, D.M., Greenberg, A.H., and Zhong, G., (1998) Inhibition of apoptosis in chlamydia-infected cells: blockade of mitochondrial cytochrome $c$ release and caspase activation. $J$ Exp Med 187: 487-496.

Galan, J.E. (2001) Salmonella interactions with host cells: type III secretion at work. Annu Rev Cell Dev Biol 17: 5386.

Gobin, S.J., van Zutphen, M., Westerheide, S.D., Boss, J.M., and van den Elsen, P.J., (2001) The MHC-specific enhanceosome and its role in MHC class I and beta (2)microglobulin gene transactivation. J Immunol 167: 51755184.

Hammerschlag, M.R., Chirgwin, K., Roblin, P.M., Gelling, M., Dumornay, W., Mandel, L., Smith, P., and Schachter, J. (1992) Persistent infection with Chlamydia pneumoniae following acute respiratory illness. Clin Infect Dis 14: 178-182.

Jendro, M.C., Deutsch, T., Korber, B., Kohler, L., Kuipers, J.G., Krausse-Opatz, B., et al. (2000) Infection of human monocyte-derived macrophages with Chlamydia trachomatis induces apoptosis of T cells: a potential mechanism for persistent infection. Infect Immun 68: 6704-6711.

Kalman, S., Mitchell, W., Marathe, R., Lammel, C., Fan, J., Hyman, R.W., et al. (1999) Comparative genomes of Chlamydia pneumoniae and C. trachomatis. Nat Genet 21: 385-389.
Kresse, A.U., Guzman, C.A., and Ebel, F. (2001) Modulation of host cell signalling by enteropathogenic and Shiga toxinproducing Escherichia coli. Int J Med Microbiol 291: 277285.

Mehta, S.J., Miller, R.D., Ramirez, J.A., and Summersgill, J.T. (1998) Inhibition of Chlamydia pneumoniae replication in HEp-2 cells by interferon-gamma: role of tryptophan catabolism. J Infect Dis 177: 1326-1331.

Naumann, M., Rudel, T., and Meyer, T.F. (1999) Host cell interactions and signalling with Neisseria gonorrhoeae. Curr Opin Microbiol 2: 62-70.

Rajalingam, K., Al Younes, H., Muller, A., Meyer, T.F., Szczepek, A.J., and Rudel, T. (2001) Epithelial cells infected with Chlamydophila pneumoniae (Chlamydia pneumoniae) are resistant to apoptosis. Infect Immun 69: 7880-7888.

Raulston, J.E. (1997) Response of Chlamydia trachomatis serovar $\mathrm{E}$ to iron restriction in vitro and evidence for ironregulated chlamydial proteins. Infect Immun 65: 45394547.

Reith, W., and Mach, B. (2001) The bare lymphocyte syndrome and the regulation of MHC expression. Annu Rev Immunol 19: 331-373.

Shaw, A.C., Vandahl, B.B., Larsen, M.R., Roepstorff, P., Gevaert, K., Vandekerckhove, J., Christiansen, G., and Birkelund, S. (2002) Characterization of a secreted Chlamydia protease. Cell Microbiol 4: 411-424.

Sullivan, J.L., and Weinberg, E.D. (1999) Iron and the role of Chlamydia pneumoniae in heart disease. Emerg Infect Dis 5: 724-726.

Vandahl, B.B., Birkelund, S., Demol, H., Hoorelbeke, B., Christiansen, G., Vandekerckhove, J., and Gevaert, K. (2001) Proteome analysis of the Chlamydia pneumoniae elementary body. Electrophoresis 22: 1204-1223.

Zhong, G., Fan, T., and Liu, L., (1999) Chlamydia inhibits interferon gamma-inducible major histocompatibility complex class II expression by degradation of upstream stimulatory factor 1. J Exp Med 189: 1931-1938.

Zhong, G., Liu, L., Fan, T., Fan, P., and Ji, H., (2000) Degradation of transcription factor RFX5 during the inhibition of both constitutive and interferon gammainducible major histocompatibility complex class । expression in chlamydia-infected cells. J Exp Med 191: 1525-1534.

Zhong, G., Fan, P., Ji, H., Dong, F., and Huang, Y., (2001) Identification of a chlamydial protease-like activity factor responsible for the degradation of host transcription factors. J Exp Med 193: 935-942. 